\title{
Research Paper: Investigation of Acoustic Characteristics of Speech Motor Control in Children Who Stutter and Children Who Do Not Stutter
}

\author{
*Fatemeh Fekar Gharamaleki ${ }^{1}$, Mohammad Rahim Shahbodaghi ${ }^{1}$, Ali Jahan ${ }^{2}$, Shohre Jalayi ${ }^{3}$
}

1. Department of Speech Therapy, School of Rehabilitation, Tehran University of Medical Sciences, Tehran, Iran. 2. Department of Speech Therapy, School of Rehabilitation, Tabriz University of Medical Sciences, Tabriz, Iran.

3. Department of Physiotherapy, School of Rehabilitation, Tehran University of Medical Sciences, Tehran, Iran.

ditation: Fekar Gharamaleki F, Shahbodaghi MR, Jahan A, Jalayi Sh. [Investigation of Acoustic Characteristics of Speech Motor Control in Children Who Stutter and Children Who Do Not Stutter (Persian)]. Archives of Rehabilitation. 2016; 17(3): 232-243. http://dx.doi.org/10.21859/jrehab-1703232

: http://dx.doi.org/10.21859/jrehab-1703232

Received: 19 Dec. 2015 Accepted: 02 Apr. 2016

Keywords: Speech motor control, Stuttering, Acoustic features, Fundamental frequency, Voice onset time

\section{ABSTRACT}

Objective Stuttering is a developmental disorder of speech fluency with unknown causes. One of the proposed theories in this field is deficits in speech motor control that is associated with damaged control, timing, and coordination of the speech muscles. Fundamental frequency, fundamental frequency range, intensity, intensity range, and voice onset time are the most important acoustic components that are often used for indirect evaluation of physiological functions underlying the mechanisms of speech motor control. The purpose of this investigation was to compare some of the acoustic characteristics of speech motor control in children who stutter and children who do not stutter.

Materials \& Methods This research is a descriptive-analytic and cross-sectional comparative study. A total of 25 Azari-Persian bilingual boys who stutter (stutters group) and 23 Azari-Persian bilinguals and 21 Persian monolingual boys who do not stutter (non-stutters group) in the age range of 6 to 10 years participated in this study. Children participated in /a/ and /i/ vowels prolongation and carrier phrase repetition tasks for the analysis of some of their acoustic characteristics including fundamental frequency, fundamental frequency range, intensity, intensity range, and voice onset time. The PRAAT software was used for acoustic analysis. SPSS software (version 17), one-way ANOVA, and Kruskal-Wallis test were used for analyzing the data.

Results The results indicated that there were no significant differences between the stutters and non-stutters groups ( $P>0.05$ ) with respect to the acoustic features of speech motor control .

Conclusion No significant group differences were observed in all of the dependent variables reported in this study. Thus, the results of this research do not support the notion of aberrant speech motor control in children who stutter.

\section{* Corresponding Author:




\title{
بررسى ويثگى هاى أكوستيكى مربوط به كنترل حركتى كَفتار در كودكان لكنتى و غيرلكنتى
}

\author{
"فاطمه فكار قراملكى'، محمدرحيم شاهباغى'، على جهان'، شهره جلايى"
}

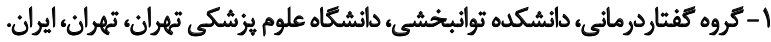

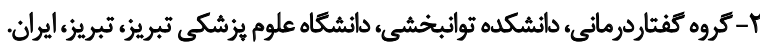

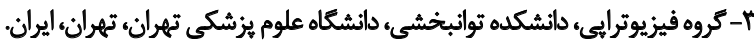

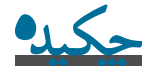

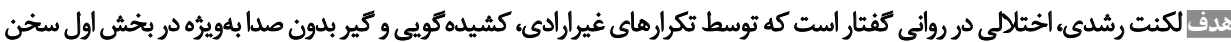

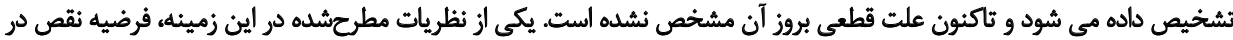

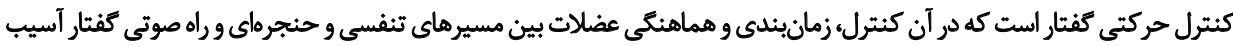

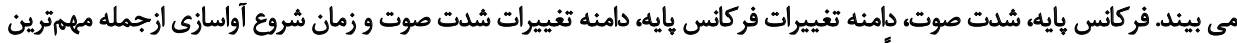

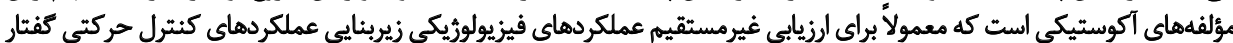

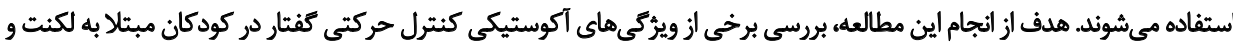

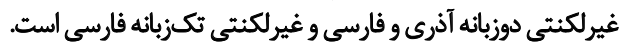

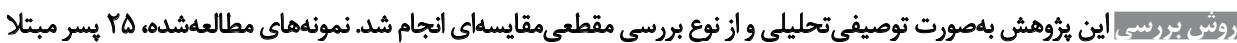

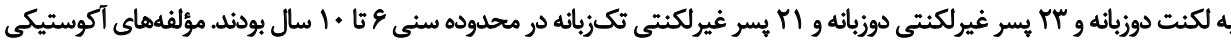

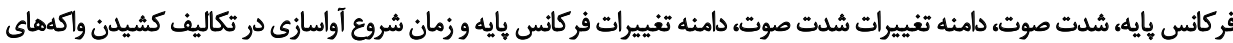

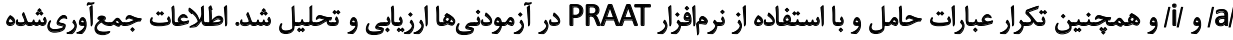
و مقادير بهدست آمده با استفاده از نسخه

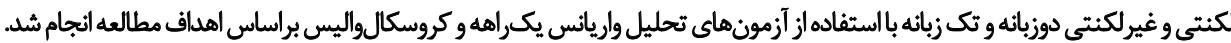

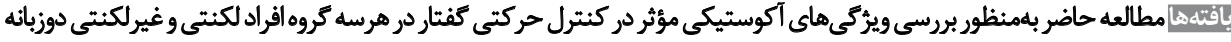

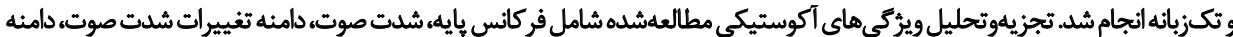

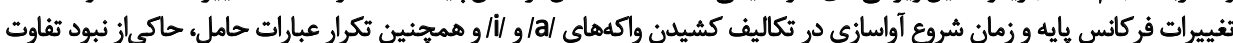

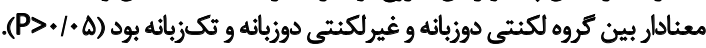

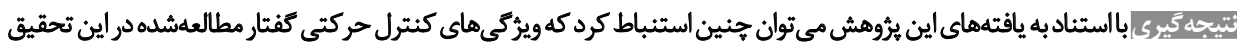

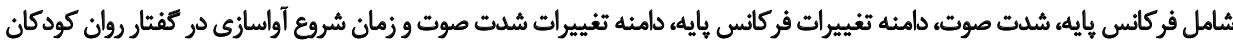

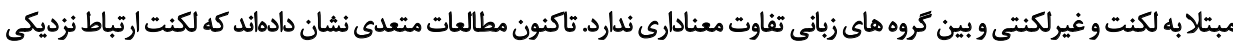

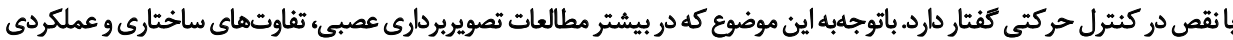

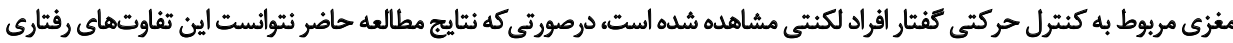

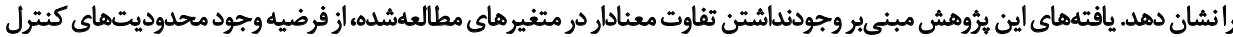

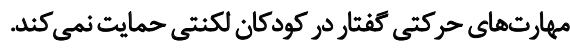

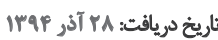

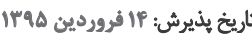

كليدوأرها:

كنترل حركتي كفتنار،

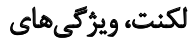

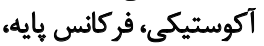
زمان شروع آواسازى فرانى بائ

حركتى كفتار است كه در آن كنترل و زمانبندى و هماهنكى

مقلمه

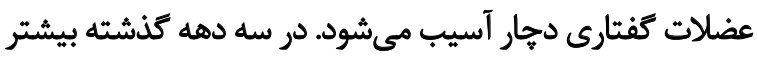

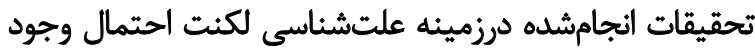

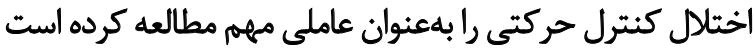

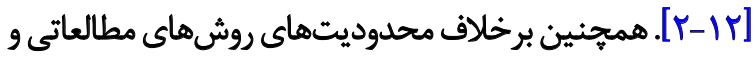

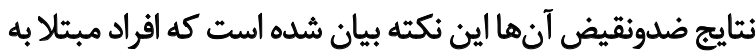

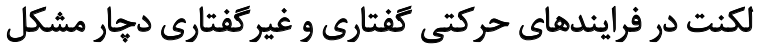

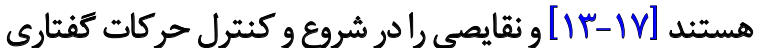

لكنت رشدى اختلالى در روانى كفتار است كه با تكرارهاى

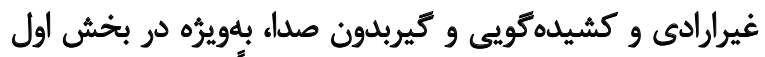

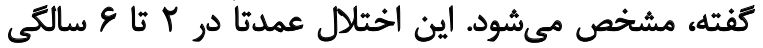

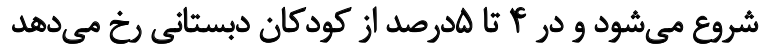

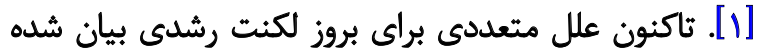

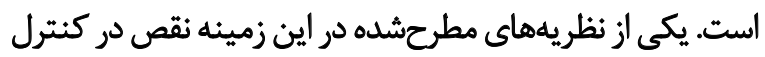

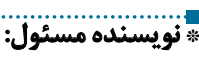
فاطمه فكار قراملكي تئيد نشانى: تبريز، خيابان وليعصر، توائير شمالى، دانشكده توانبخشى، كروه كفتاردرمانى.

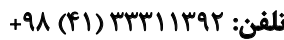
رايانامه: slp.fekar@yahoo.com 
و آكوستيك صوتى يكى از بهترين مطالعات غيرتهاجمى و عينى مئى

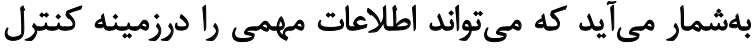
حركتى كفتار دراختيار ما قرار دهد [سبان. فركانس عايه، شدت صوت، دامئه تغييرات فركانس بايه، دامنه

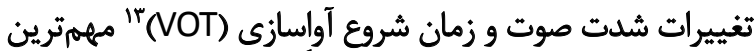

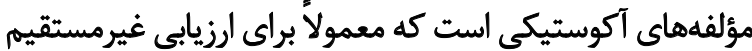

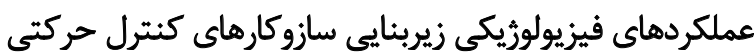

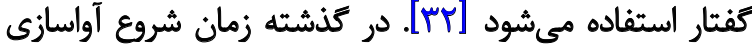

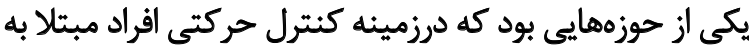

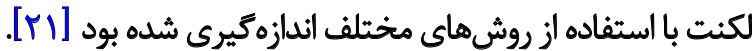

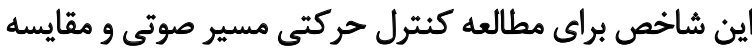

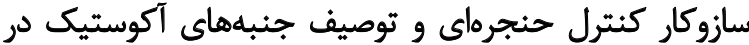

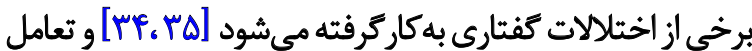

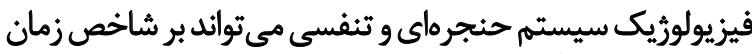
شروع آواسازى تأثير بكذارد [هبآ]

مرور مطالعات كذشته درباره زمان شروع آواسازى نتايج بىثباتى

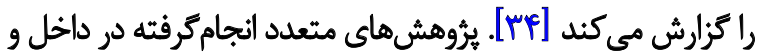

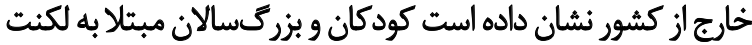

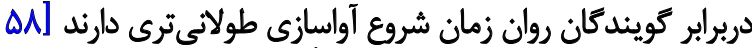

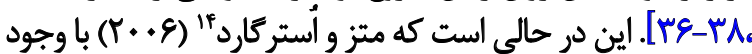

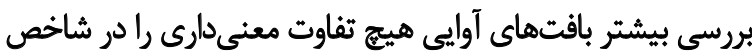

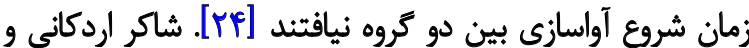

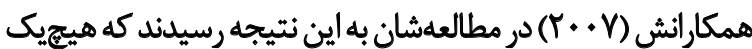

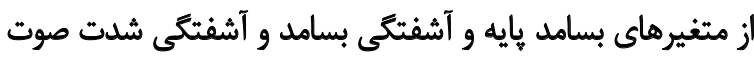

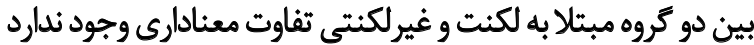

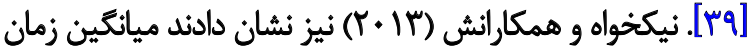

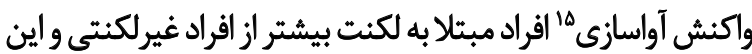

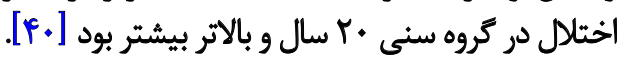

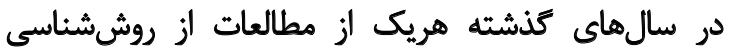

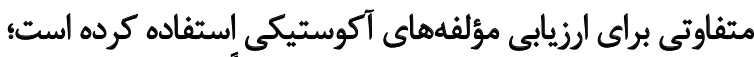

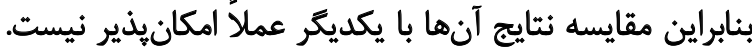

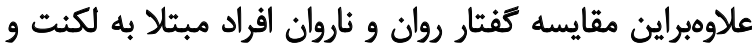

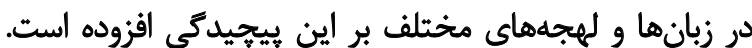

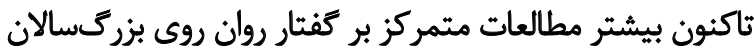

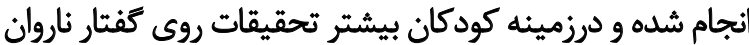

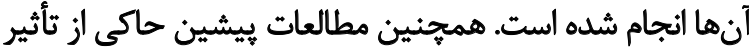

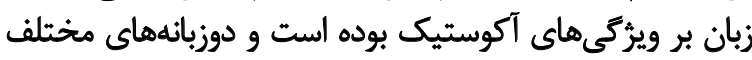

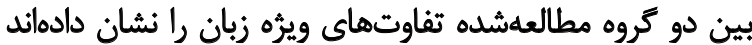

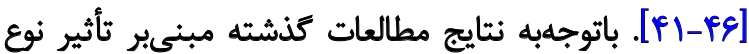

13. Voice Onset Time (VOT)

14. Metz and $\varnothing$ stergaard

15. Reaction time of phonation

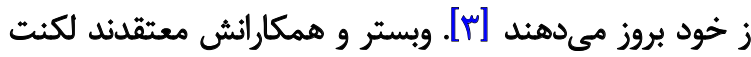

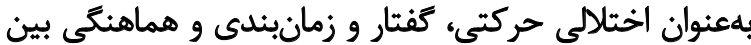

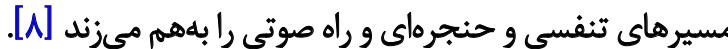
مطالعه لكنت از ديدكاه اختلال در كنترل حركتى كفتار بسيار

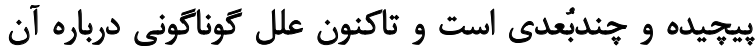

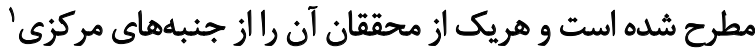

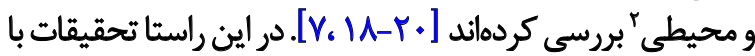

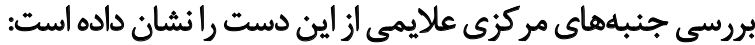

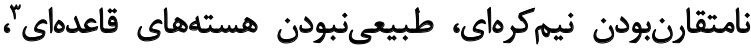

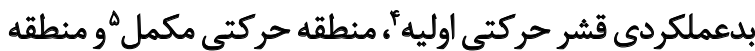

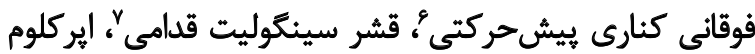

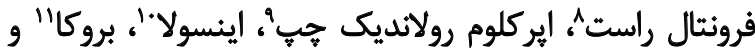

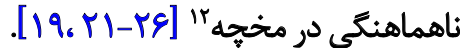

مقايسه تصويربردارى مغزى افراد مبتلابه لكنت نشان داد حين

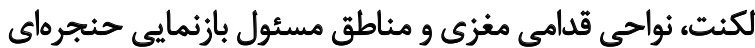

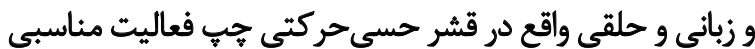

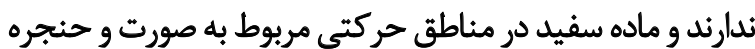

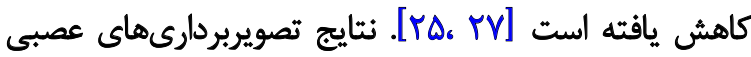

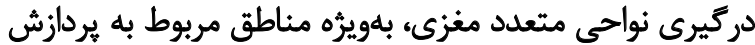

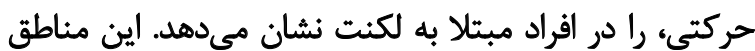

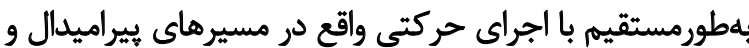

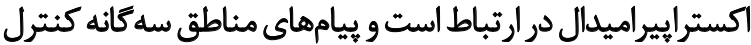

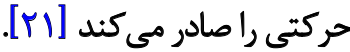

علاوهبر مشكلات مركزى عصبشتاختي، عملكرد كنترل

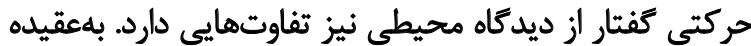

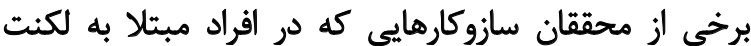

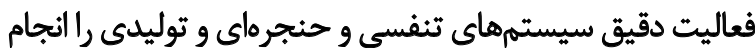

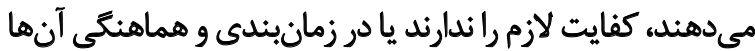

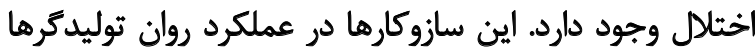

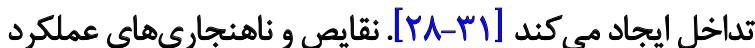

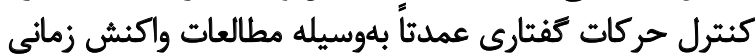

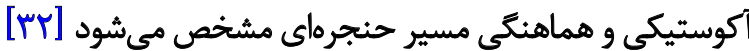

1. Central

2. Peripheral

3. Basal gangelia

4. Primary motor cortex

5. Supplementary Motor Area (SMA)

6. Superior lateral premotor area

7. Anterior cingulate cortex

8. Right frontal operculum

9. Left Rolandic operculum

10. Insula

11. Broca

12. Cerebellum 


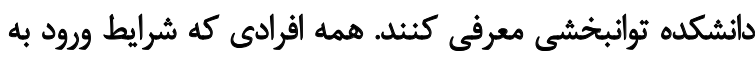

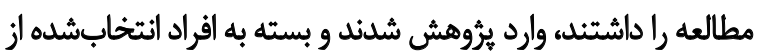

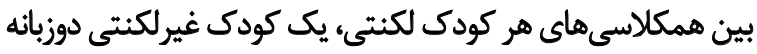

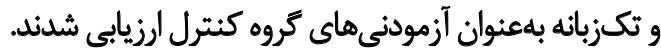

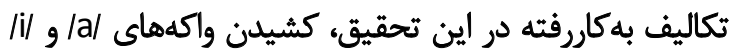

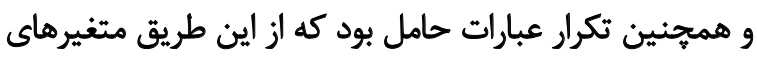

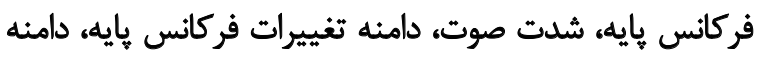

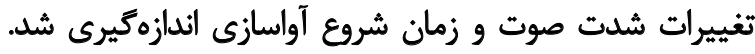

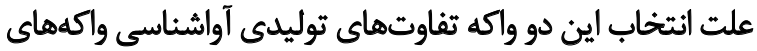

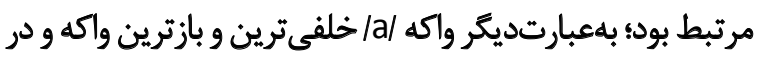

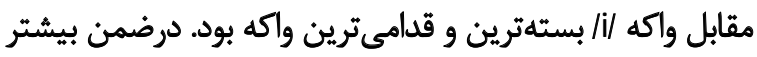

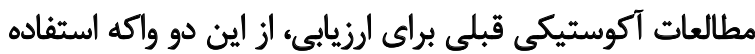

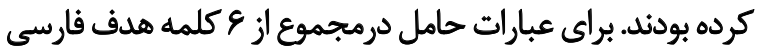

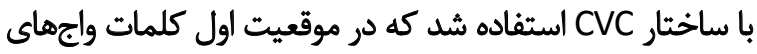

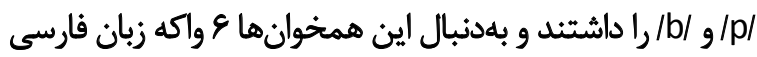
در بافت كلمه وجود داشت (ييوست شماره ال).

وجود اين همخوان ها در موقعيت اول كلمه جندين علت تحليلى ماندي

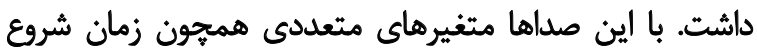

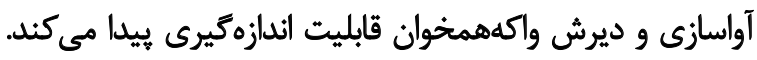

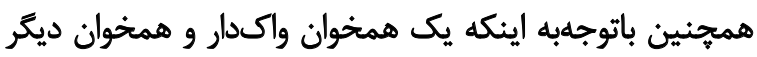

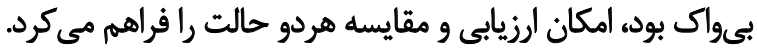

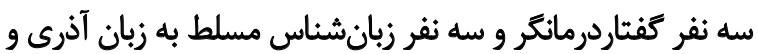

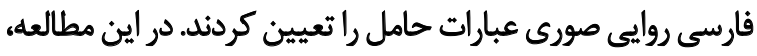

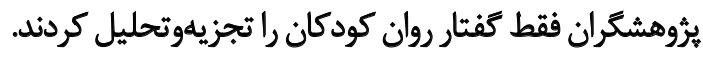

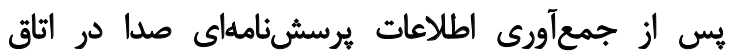

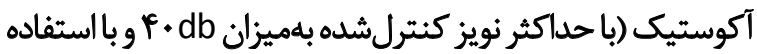

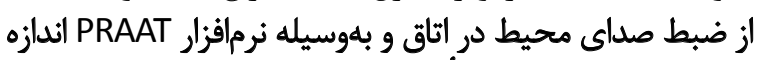

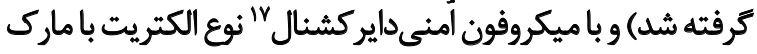

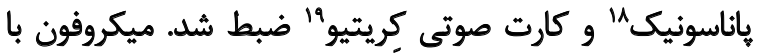

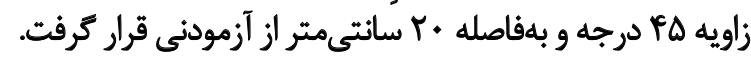

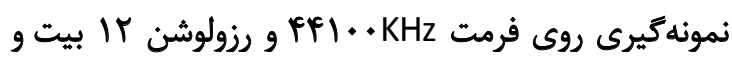

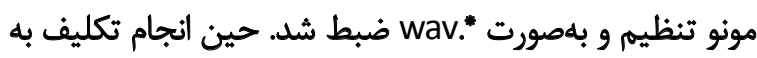

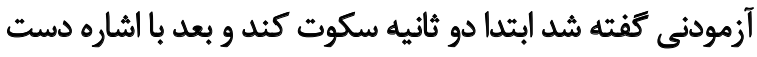

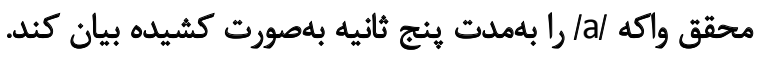

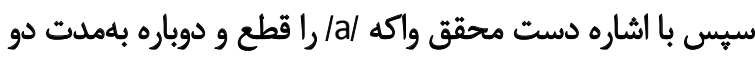

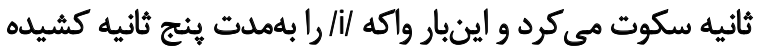

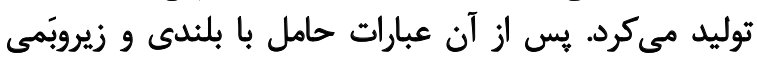

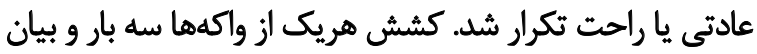

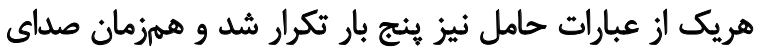

17. Omnidirectional

18. Panasonic

19. Creative

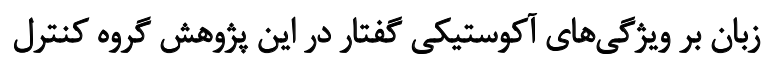

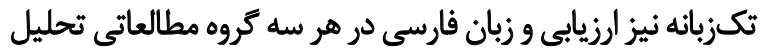

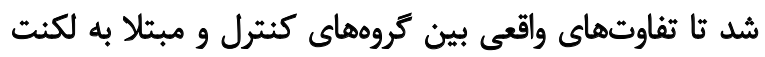

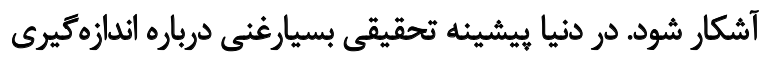

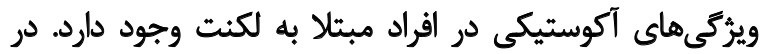

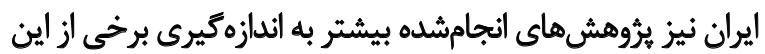

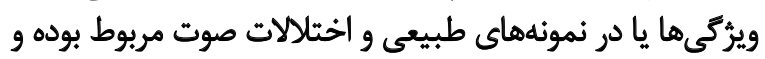

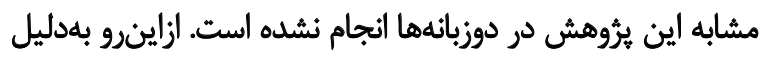

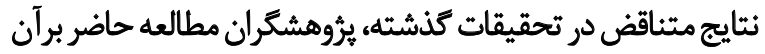

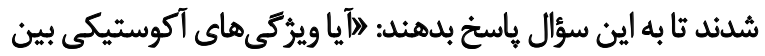

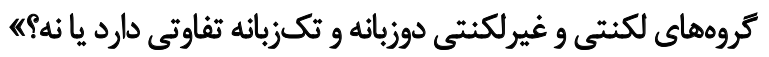

$$
\text { ووشّ برورى }
$$

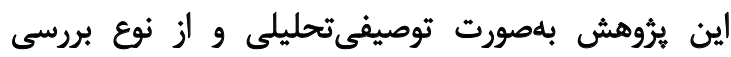

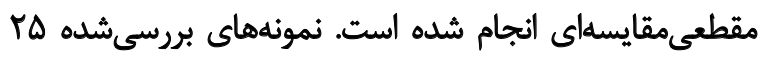

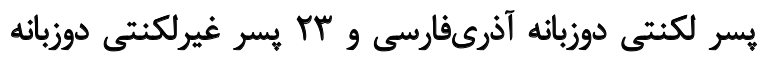

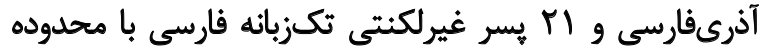

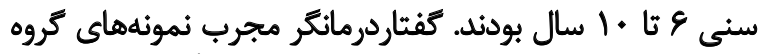

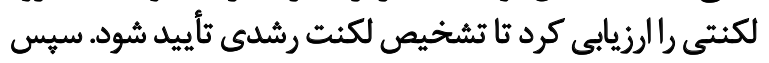

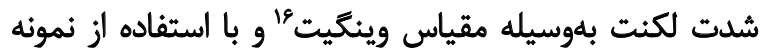

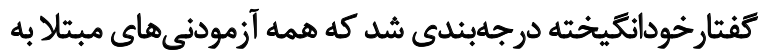
لكنت در سطح متوسط و شديد قرار كرفتند.

معيارهاى ورود به مطالعه براى كروه لكنتى شامل اين معيارها

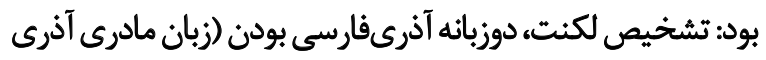

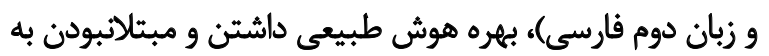

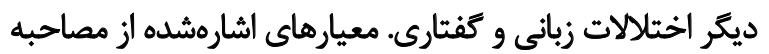

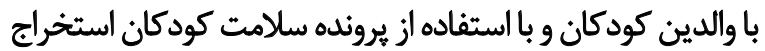

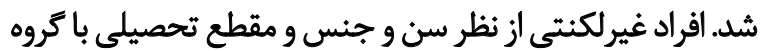

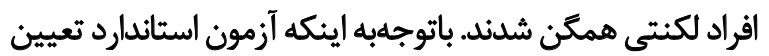

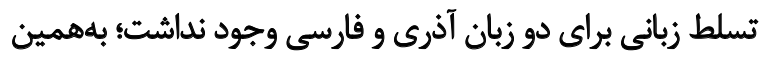

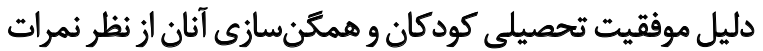
زبان فارسى و املا ملاك تسلط زبانى كودئ قرار كرفت

كودكان كروه غيرلكنتى تكزبانه بهمنظور ورود به مطالعيه

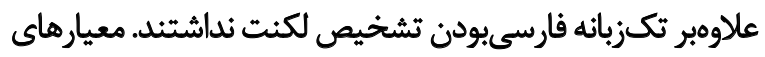

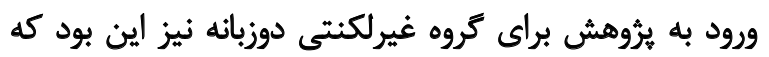

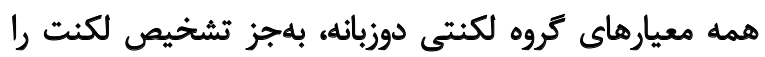

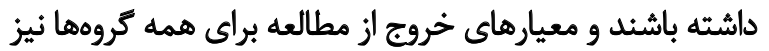
همكارىنكردن كودك بود.

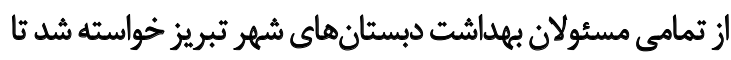

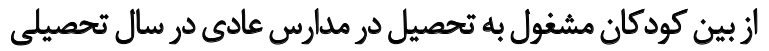

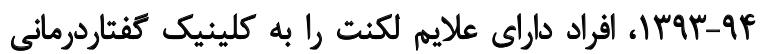

16. Wingate rate 
فركانس يايه در واكه /a//ز نظر آمارى بين سه كروه مطالعهشده

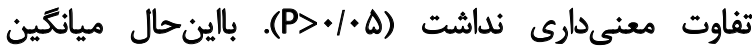

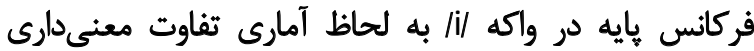

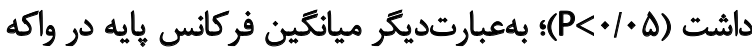

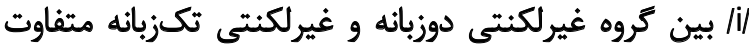

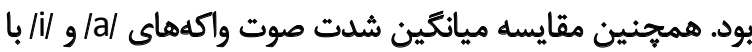

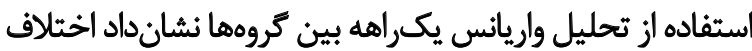

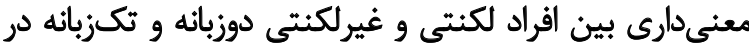
كشيدن واكه وجود نداشت (ه) (P> (P).

آزمونهاى كروسكالواليس و يومانويتنى نشان داد ميانه

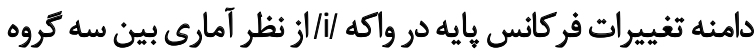

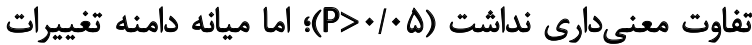

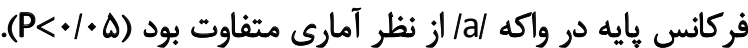

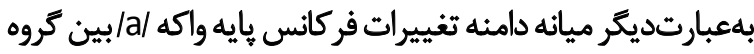

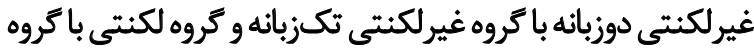

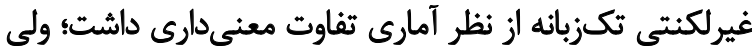

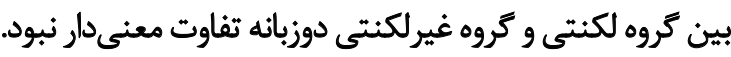

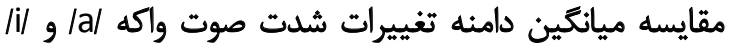

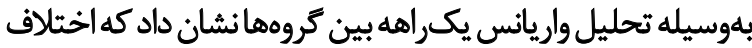

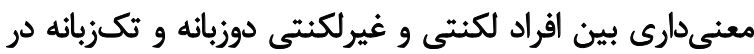

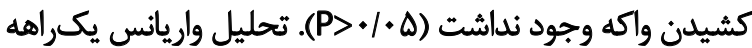

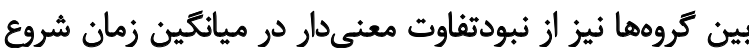

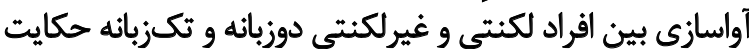

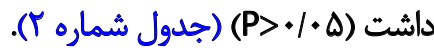

بحث

مطالعه حاضر بهمنظور بررسى ويُّكى هاى آكوستيكى مؤثر در

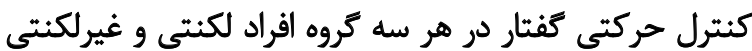

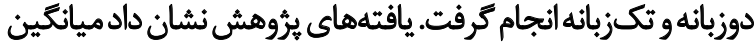

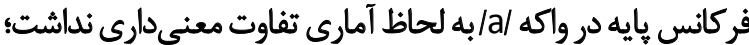

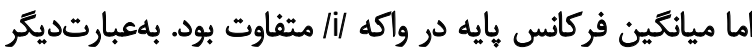

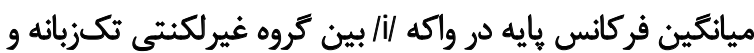

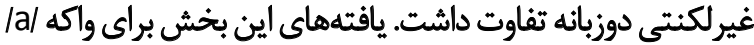

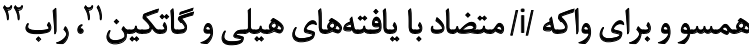

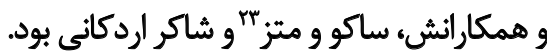

برخلاف اين مطالعه كه واكهها بهتنهايى توليد شدنده در

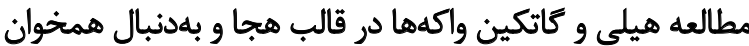

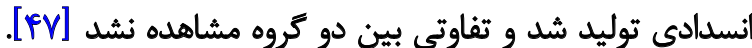

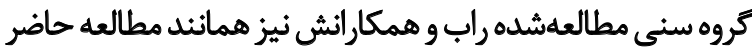

21. Healey and Gutkin

22. Robb

23. Sacco and Metz

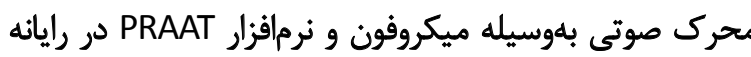

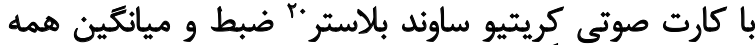

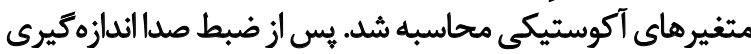

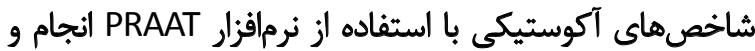

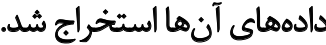

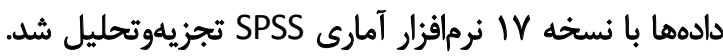

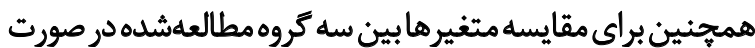

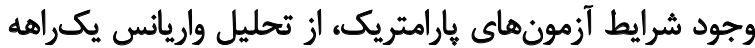

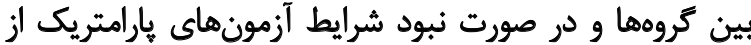

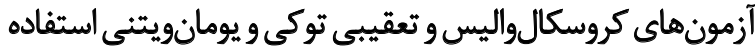

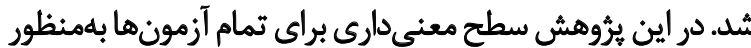

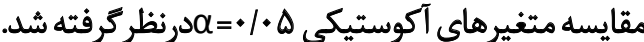

در تمامى مراحل مطالعه ملاحظات اخلاقى براساس اصول

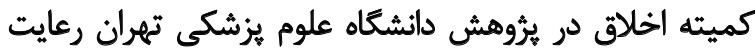

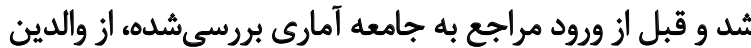

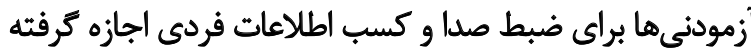

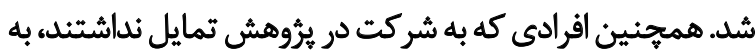

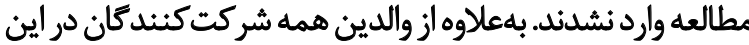
يُروهش فرم رضايتنامه كتبى دريافت و به آنها آنها اطمينان دادين

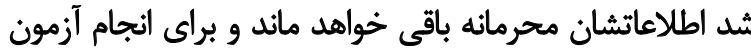

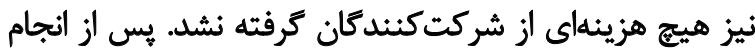

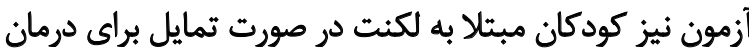

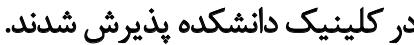

ياقتهها

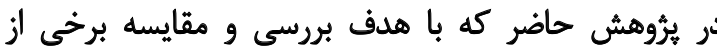

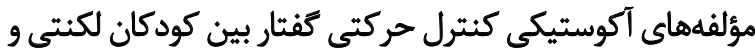

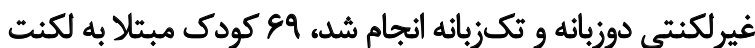

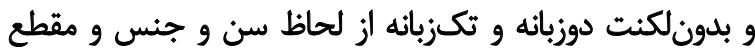
تحصيلى با كودكان گروه لكنتى مطابقت داده (جدول شئ شماره

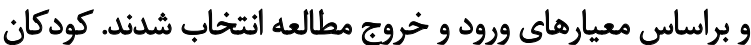

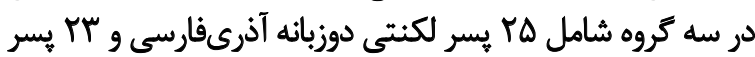

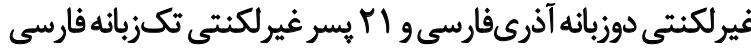

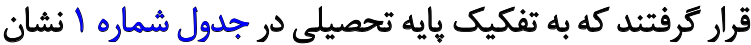

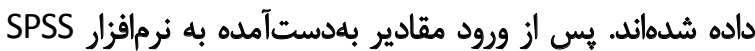

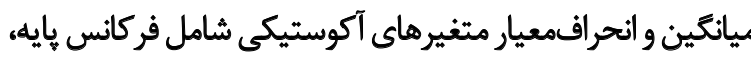

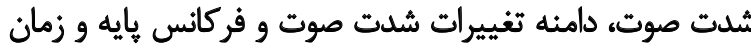

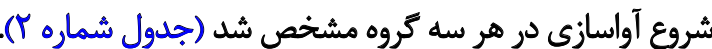
تجزيلوتحليل آمارى دادههاي بهدست آمده از مقايسه تروههاي لكنتى و غيرلكنتى دوزبانه و تكزبانه، با استفادها از تحليل وارياني يكراهه بين گروهها وآزمون تعقيبى توكى نشان دان داد كه ميانتينين

20. Creative sound blaster 
جدول الم توصيف نمونهاى كودكان لكنتى و غيرلكنتى.

\begin{tabular}{|c|c|c|c|c|c|c|}
\hline كل & نايه يُنجم & بايه جهارم & يايه سوم & بايه دوم & بايه اول & كروههاي مطالعهشده \\
\hline ro & r & r & v & 8 & $\&$ & لكنتى \\
\hline r & r & r & 8 & r & 1. & غيرلكنتى دوزبانه \\
\hline$M$ & $p$ & $\varphi$ & 8 & $r$ & p & غغيرلكتنى تكزيانه \\
\hline $9 q$ & $\wedge$ & 1. & 19 & ir & r. & 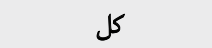 \\
\hline
\end{tabular}

جدول T. ميانكين و انحرافمعيار و نتايج آزمون آمارى متغير هاي آكوستيكي كودكان لكثتى و كودكان غيرلكنتي.

\begin{tabular}{|c|c|c|c|c|c|c|c|c|}
\hline \multirow{2}{*}{ مقدار احتمال } & \multicolumn{2}{|c|}{ لكتى تكزبانه } & \multicolumn{2}{|c|}{ غيرلكتتى دوزبانه } & \multicolumn{2}{|c|}{ ل كتثى } & & \multirow{2}{*}{ متغير مستقل/كروه } \\
\hline & مياتكين & انحرافمعيار & مياتعين & انحرافمعيار & مياتيّين & انحراقشعيار & & \\
\hline.$/ M r$ & $r / / \Delta$ & $\pi r \Delta / \mu$ & $r A / / Q$ & rot/q. & m/qD & $M E q / \Delta S$ & /a/ & \\
\hline$\%$ & $r \Delta / v E$ & MeT/AP & $r q / T V$ & TEVIFA & $r \cdot 1 . V$ & $r \Delta \cdot / M$ & /i/ & فركائ بايه در كشيدن واكه \\
\hline.$/ M^{e}$ & $r / r q$ & VN/T & $r / T r$ & VNAV & r/M & VNII & /a/ & \\
\hline.$/ v$ & $r / \cdot 9$ & $W / \& \Lambda$ & V/u & $V 9 / \% \Delta$ & $r / 9 \Lambda$ & $v \& M$ & /i/ & شدث صوت در كثيلن واكه \\
\hline.$/ .48$ & $r \cdot / \Lambda r$ & $r / u$ & $\Delta T / N$ & $q \vee / M^{\circ}$ & rV/ar & AV/Aq & /a/ & دامنه تغييرات فركانس هايه در \\
\hline.$/ 4 r$ & $r \cdot / r$ & $M / M$ & 198. & r & $\pi / A \mid$ & $\pi / \Delta \Delta$ & /i/ & كشيدن واكله \\
\hline.$/ M$ & VeV & $\Delta / \cdot r$ & $1 / \pi f$ & $\Delta / \pi r$ & $r / \Delta 1$ & s/.r & /a/ & دامنه تغييرات شدت صوت در \\
\hline .1 .0 & $1 / 19$ & $\Delta / \cdot r$ & $r / 48$ & 81.9 & $r / N$ & $g / N A$ & /i/ & كشيدن واكه \\
\hline.$/ / v$ & $.1 \cdot 11$ & $.1 \cdot v+$ & $.1 \cdot 4$ & $* 1+n$ & .1 .18 & .1 .94 & /pak/ & \\
\hline.$/ r$ & .1 .4$. & $.1 .4 r$ & $.1 \cdot 11$ & $.1 \circ \Lambda \Delta$ & .1 .5 & .1 .94 & /puch/ & \\
\hline.$/ \mathrm{NA}$ & .1 .19 & 1.94 & .1 .18 & .1 .98 &.$/ . \mathrm{r}$ & .1 .09 & /pæs/ & \\
\hline.$/ 4$ & $.1 \cdot+r$ & .1 .14 &.$/ .1 \pi r$ &.$/ * 1 Y$ & $.1 * \cdot 1$ & .1 .10 & /beh/ & زمان شروع آواسازى در كلمات هدف \\
\hline.$/ v 9$ &.$\%$ &.$/ .14$ & $.1 .+4$ & $.1+16$ & $* 1 * * 1$ &.$/ * 1 f$ & //bam & \\
\hline.$|A|$ & .10 .8 &.$/ 4 r$ & .1 .00 & $1.4 r$ & .1 .08 & $.1 .4 r$ & /bud/ & \\
\hline
\end{tabular}

توانبخننى

يافتههاي فلك مَ و همكارانش و ساكو و متز مشابه بود. در مطالعه

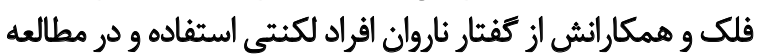

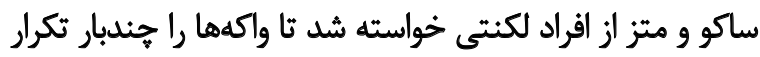

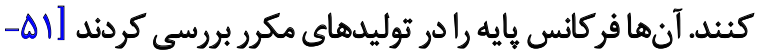

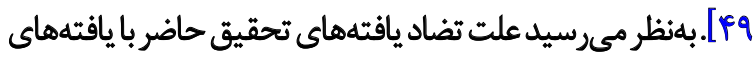

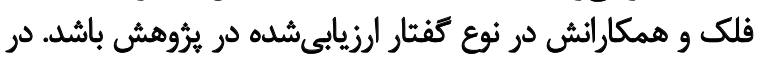

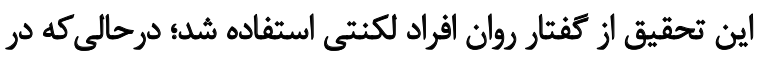

دانش آموزان يسر بودند. در اين گروه سنى نيز تفاوت معنى دارى

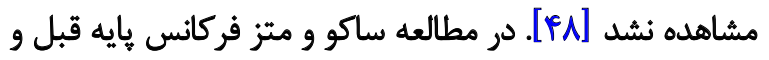
بعد از درمان بررسى شد كه نتايج حاكى ازئ اين بود كه درمان

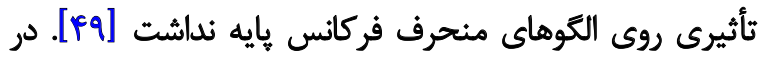

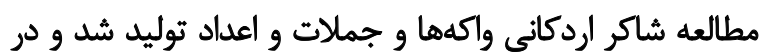

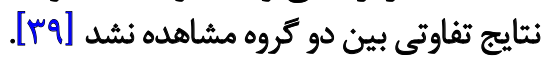
يافتهاي اين بخش براي واكه /a/ متفاوت و براى واكه /i/ با بال

24. Flack 
نتايج بهدستآمده نشان داد ميانكين شروع زمان آواسازى بين

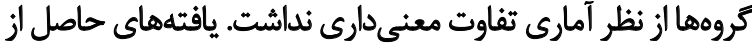

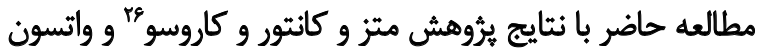

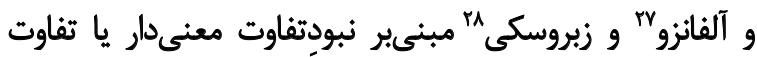

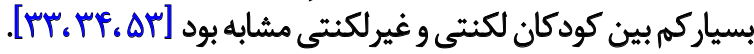

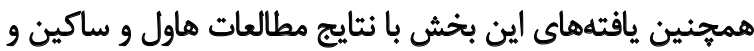

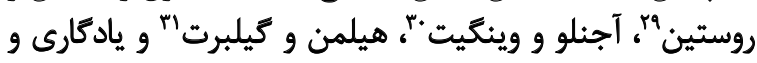

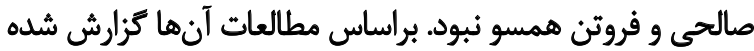

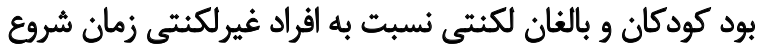

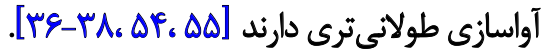
مطالعه سيه و زبروسكى بٓنيز نشان داد زمان شروع آواسازى

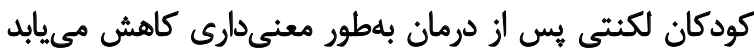

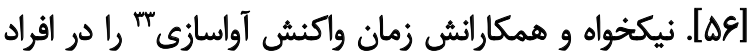

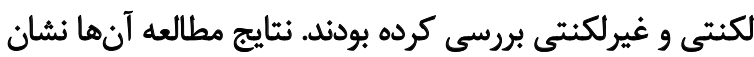

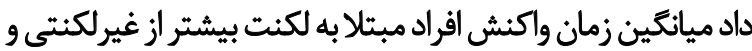

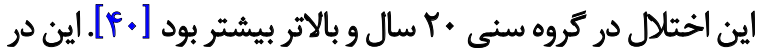

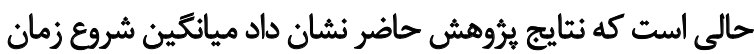

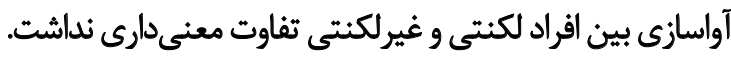
تجزيهوتحليل ويرُكىهاى آكوستيكى كه در كنترل حركتى

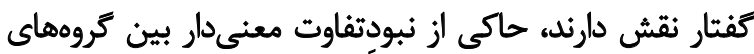

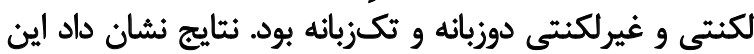

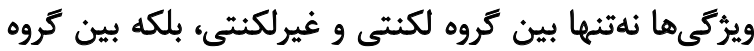

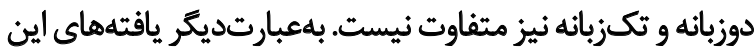

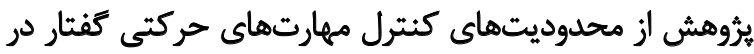

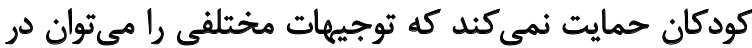

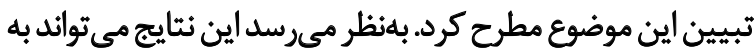

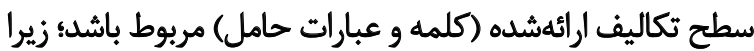

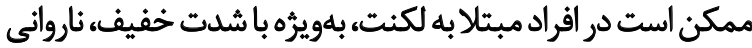

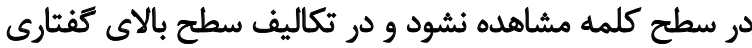

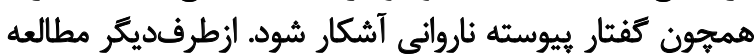

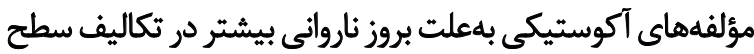

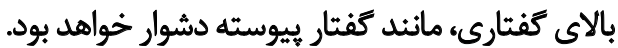

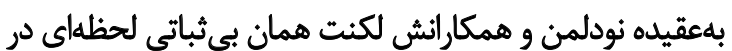

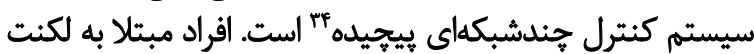

26. Metz, Conture and Caruso

27. Watson and Alphonso

28. Zebrowski

29. Howell, Sackin and Rustin

30. Agnello and Wingate

31. Hillman and Gilbert

32. Sim and Zebrowski

33. Phonatory reaction time

34. Complex multiloop control system
مطالعه فلك و همكارانش كفتار ناروان آنها بهكاررفت.

نتايج بهدست آمده نشان داد كه ميانكين شدت صوت در واكهها

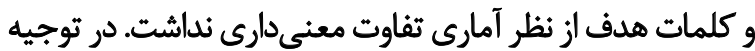

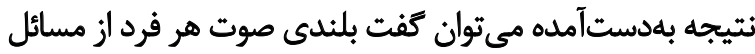

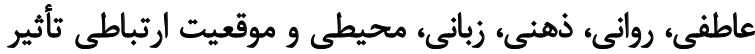

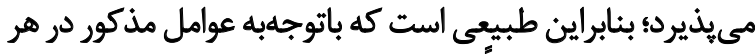

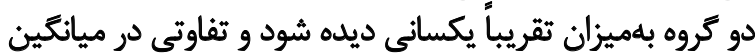
شدت صوت وجود نداشته باشد.

تجزيلوتحليل آمارى دادههاى يُورهش حاضر نشان داد

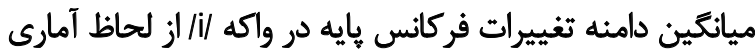

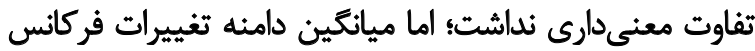

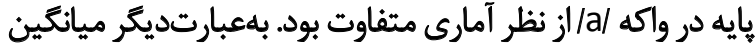

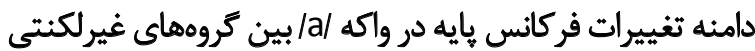

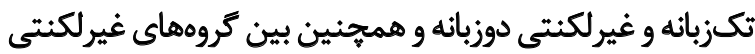

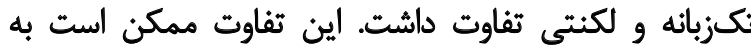

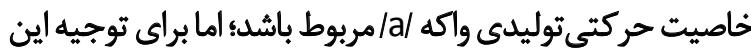
يافته نياز به مطالعه بيشترى است.

تحقيقاتيى انجامشده درزمينه ميانكين دامنه تغييرات فركانس

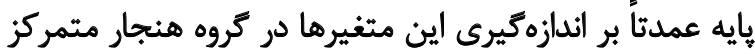

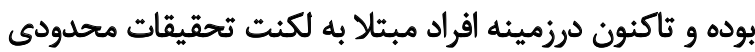

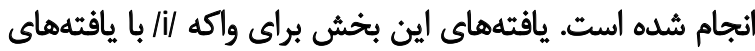

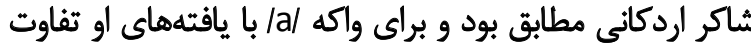

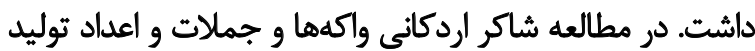

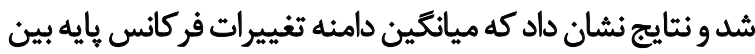

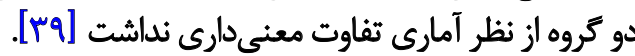

همجئين تجزيهوتحليل آمارى دادههاى يُروهش حاضر نشان

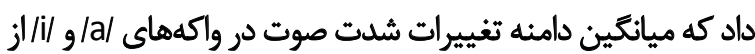

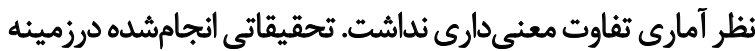

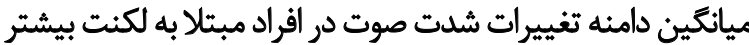

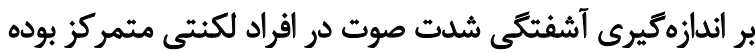

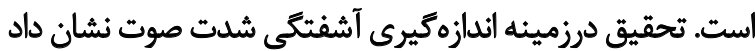

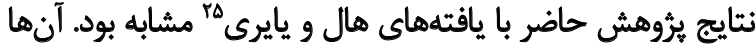

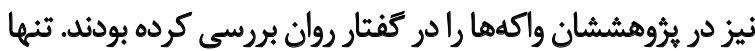

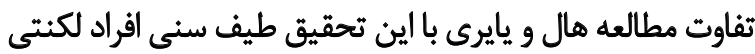

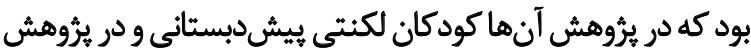

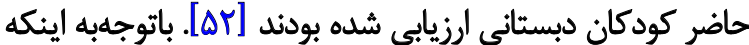

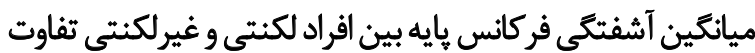

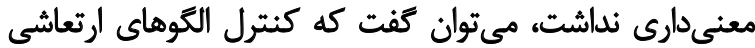

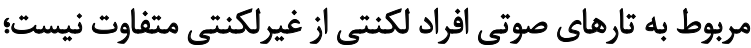

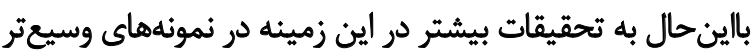

$$
\text { نياز است. }
$$

25. Hall and Yair 
يرؤوهش روى يك جنس و درنظرئكرفتن كفتار ناروان آزمودنىها

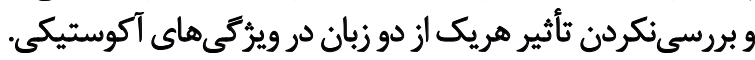

Dosteinis

باتوجهبه نتايج مطالعه حاضر و مطالعات قبلى ييشنهاد مي كئود

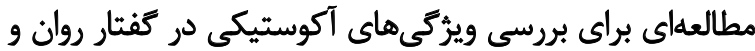

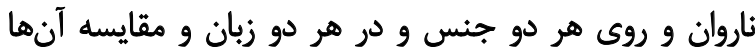

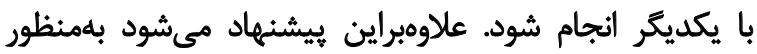

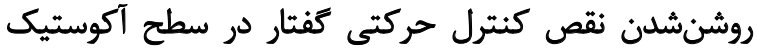

$$
\text { يثوهش هاى بيشترى انجام شود. }
$$

$$
\text { تشكر و قدردانى }
$$

اين مقاله مستخرج از باياننامه كارشناسىارشد خانم فاطمانه

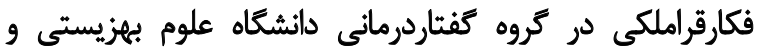
توانبخشى تهران است

هميشه شواهد ناروانى را نشان نمى دهند أنها زمانى علايمى از

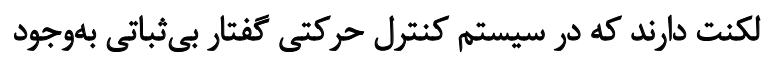

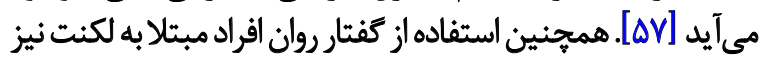

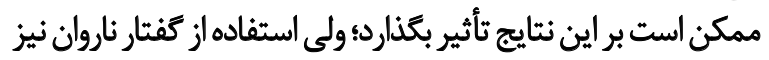

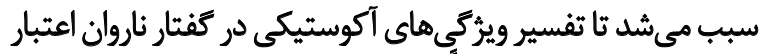
كافى را نداشته باشد و الزامأ از ناروانى كفتار تأثير بذئيرد.

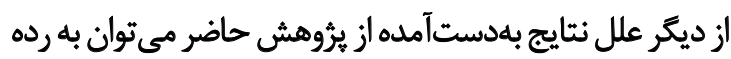

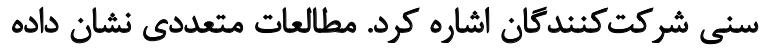

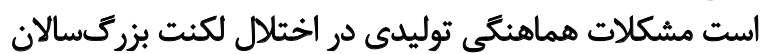

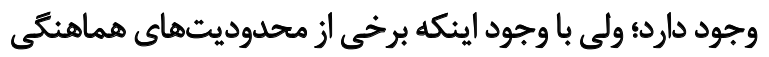

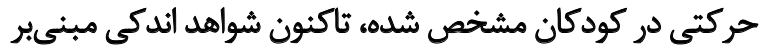
مشكلات مشابه بزركسالان در كودكان به اثبات رسيده است.

$$
\text { نتيجليَّيرى }
$$

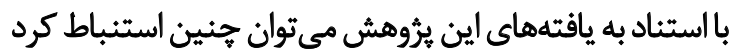

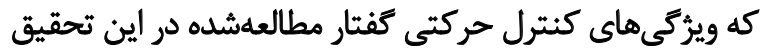

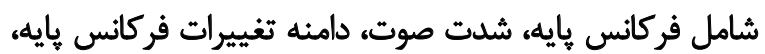

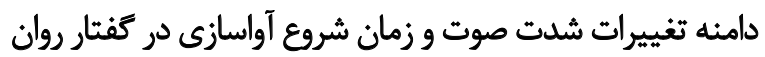

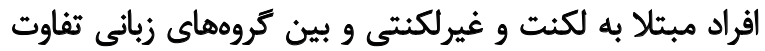

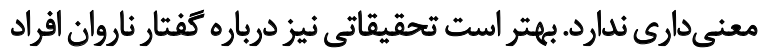

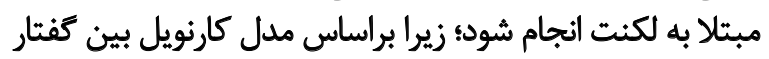

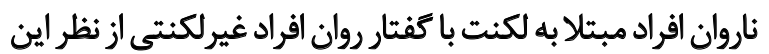

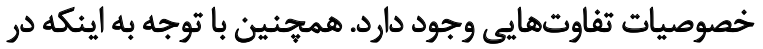

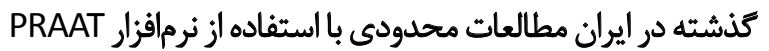

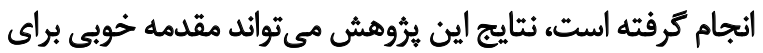

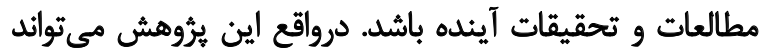

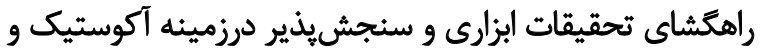

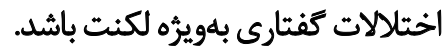

lovenges

مهمترين محدوديتهاى مطالعه حاضر عبارت بود از: انجام

يبيوست ا ـ فهرست عبارات حامل.

"إبه يك كلمه فارسى است.

"ابامه يك كلمه فارسى است.

(بوده يك كلمه فارسى است.

\section{" إياكى يك كلمه فارسى است.}

" ايوج" يك كلمه فارسى است.

" إيس يك كلمه فارسى است.

توانبخننى

ييوست ץ ـ واجنكارى عبارات حامل.

/ beh yek kalameye farsi æst /

/ bam yek kalameye farsi æst /

/ bud yek kalameye farsi æst /
/ pak yek kalameye farsi æst /

/ putf yek kalameye farsi æst /

/ pæs yek kalameye farsi æst /

توانبخننى 


\section{References}

[1] Sato, Y, Mori, K, Koizumi, T, Minagawa-Kawai, Y, Tanaka, A, Ozawa, E, Mazuka, R. Functional lateralization of speech processing in adults and children who stutter. Frontiers in psychology. 2011; 2. doi: 10.3389/fpsyg.2011.00070

[2] Ingham RJ. On learning from speech-motor control research on stuttering. In: Cordes AK, Ingham RJ, editors. Treatment Efficacy for Stuttering: A Search for Empirical Bases. San Diego: Singular Publishing Group; 1998, p. 67-101.

[3] Peters HM, Hulstijn W, van Lieshout PH. Recent developments in speech motor research into stuttering. Folia Phoniatrica et Logopaedica. 2000; 52(1-3):103-19. doi: 10.1159/000021518

[4] Postma A, Kolk H, Povel DJ. Speech planning and execution in stutterers. Journal of Fluency Disorders. 1990; 15(1):49-59. doi: 10.1016/0094-730x(90)90032-n

[5] Bloodstein O. A handbook on stuttering. Massachusetts: Cengage Learning; 1987.

[6] Ludlow C, Siren K, Zikira M. Speech production learning in adults with chronic developmental stuttering. Speech production. In: Hujstijn W, Peters HFM, van Lieshout PH, editors. Speech Production: Motor Control, Brain Research and Fluency Disorders. Amsterdam: Elsevier Science Publishers; 1997, p. 212-29.

[7] Jones RD, White AJ, Lawson KH, Anderson TJ. Visuoperceptual and visuomotor deficits in developmental stutterers: an exploratory study. Human Movement Science. 2002; 21(5):60319. doi: 10.1016/s0167-9457(02)00165-3

[8] van Lieshout PH, Hulstijn WO, Peters HF. Searching for the weak link in the speech production chain of people who stutter: a motor skill approach. In: Maassen B, Kent R, Peters HFM, van Lieshout P, Hulstijn W, editors. Speech Motor Control in Normal and Disordered Speech. Oxford: Oxford University Press; 2004, p. 313-35.

[9] Duffy JR. Motor speech disorders: substrates, differential diagnosis and management. $2^{\text {nd }}$ ed. St Louis: Mosby Pub; 2005.

[10] Smits-Bandstra S, Luc F, Saint-Cyr JA. Speech and nonspeech sequence skill learning in adults who stutter. Journal of Fluency Disorders. 2006; 31(2):116-36. doi: 10.1016/j. jfludis.2006.04.003

[11] van Lieshout PH, Hulstijn W, Peters HF. From planning to articulation in speech production what differentiates a person who stutters from a person who does not stutter? Journal of Speech, Language, and Hearing Research. 1996; 39(3):546-64. doi: 10.1044/jshr.3903.546

[12] van Lieshout PH, Hulstijn W, Peters HF. Speech production in people who stutter testing the motor plan assembly hypothesis. Journal of Speech, Language, and Hearing Research. 1996; 39(1):76-92. doi: 10.1044/jshr.3901.76

[13] Adams MR, Hayden P. The ability of stutterers and nonstutterers to initiate and terminate phonation during production of an isolated vowel. Journal of Speech, Language, and Hearing Research. 1976; 19(2):290-6. doi: 10.1044/jshr.1902.290
[14] Maske-Cash WS, Curlee RF. Effect of utterance length and meaningfulness on the speech initiation times of children who stutter and children who do not stutter. Journal of Speech, Language, and Hearing Research. 1995; 38(1):18-25. doi: 10.1044/jshr.3801.18

[15] Max L, Caruso AJ, Gracco VL. Kinematic analyses of speech, orofacial nonspeech, and finger movements in stuttering and nonstuttering adults. Journal of Speech, Language, and Hearing Research. 2003; 46(1):215-32. doi: 10.1044/10924388(2003/017)

[16] Packman A, Code C, Onslow M. On the cause of stuttering: integrating theory with brain and behavioral research. Journal of Neurolinguistics. 2007; 20(5):353-62. doi: 10.1016/j.jneuroling.2006.11.001

[17] Smits-Bandstra S, de Nil L, Rochon E. The transition to increased automaticity during finger sequence learning in adult males who stutter. Journal of Fluency Disorders. 2006; 31(1):22-42. doi: 10.1016/j.jfludis.2005.11.004

[18] Watkins KE, Smith SM, Davis S, Howell P. Structural and functional abnormalities of the motor system in developmental stuttering. Brain. 2008; 131(1):50-9. doi: 10.1093/brain/ awm241

[19] Alm PA. Stuttering and sensory gating: a study of acoustic startle prepulse inhibition. Brain and Language. 2006; 97(3):317-21. doi: 10.1016/j.bandl.2005.12.001

[20] Molt LF. The basal ganglia's possible role in stuttering: an examination of similarities between stuttering, Tourette Syndrome, Dystonia, and other neurological-based disorders of movement [Internet]. Proceedings of the $2^{\text {nd }}$ International Stuttering Awarness Day. 1999. Available from: https://www. mnsu.edu/comdis/isad2/papers/molt2.html

[21] Ward D. Stuttering and cluttering: frameworks for understanding and treatment. Routledge: Taylor \& Francis; 2008.

[22] Sommer M, Koch MA, Paulus W, Weiller C, Büchel C. Disconnection of speech-relevant brain areas in persistent developmental stuttering. The Lancet. 2002; 360(9330):380-3. doi: 10.1016/s0140-6736(02)09610-1

[23] Chang SE, Kenney MK, Loucks TM, Ludlow CL. Brain activation abnormalities during speech and non-speech in stuttering speakers. Neuroimage. 2009; 46(1):201-12. doi: 10.1016/j. neuroimage.2009.01.066

[24] Mertz LB, Østergaard JR. Neurological aspects of stuttering. Ugeskrift for Laeger. 2006; 168(37):3109-13.

[25] Braun AR, Varga M, Stager S, Schulz G, Selbie S, Maisog JM, et al. Altered patterns of cerebral activity during speech and language production in developmental stuttering. $\mathrm{An}_{2}(15) \mathrm{O}$ positron emission tomography study. Brain. 1997; 120(5):76184. doi: 10.1093/brain/120.5.761

[26] Craig-McQuaide A, Akram H, Zrinzo L, Tripoliti E. A review of brain circuitries involved in stuttering. Frontiers in Human Neuroscience. 2013; 8:884-884. doi: 10.3389/fnhum.2014.00884

[27] Chang SE, Erickson KI, Ambrose NG, Hasegawa-Johnson MA, Ludlow CL. Brain anatomy differences in childhood stut- 
tering. Neuroimage. 2008; 39(3):1333-44. doi: 10.1016/j.neuroimage.2007.09.067

[28] Adams MR. A physiologic and aerodynamic interpretation of fluent and stuttered speech. Journal of Fluency Disorders. 1974; 1(1):35-47. doi: 10.1016/s0094-730x(74)80005-7

[29] Kent, RD. Stuttering as a temporal programming disorder. In: Curlee RF, Perkins WH, editors. Nature and Treatment of Stuttering: New Directions. San Diego: Colledge-Hill Press; 1984, p. 283-301.

[30] van Riper C. The nature of stuttering. Illinois: Waveland Pub; 1971.

[31] Zimmermann G. Stuttering: a disorder of movement. Journal of Speech, Language, and Hearing Research. 1980; 23(1):12236. doi: $10.1044 /$ jshr.2301.122

[32] Peters HF, Hulstijn W, Starkweather CW. Acoustic and physiological reaction times of stutterers and nonstutterers. Journal of Speech, Language, and Hearing Research. 1989; 32(3):66880. doi: $10.1044 /$ jshr.3203.668

[33] Zebrowski PM, Conture EG, Cudahy EA. Acoustic analysis of young stutterers' fluency: Preliminary observations. Journal of Fluency Disorders. 1985; 10(3):173-92. doi: 10.1016/0094730x(85)90009-9

[34] Watson BC, Alfonso PJ. A comparison of LRT and VOT values between stutterers and nonstutterers. Journal of Fluency Disorders. 1982; 7(2):219-41. doi: 10.1016/0094730x (82)90010-9

[35] Auzou P, Ozsancak C, Morris RJ, Jan M, Eustache F, Hannequin $\mathrm{D}$. Voice onset time in aphasia, apraxia of speech and dysarthria: a review. Clinical Linguistics \& Phonetics. 2000; 14(2):131-50. doi: 10.1080/026992000298878

[36] Yadegari F, Salehi A. [Diffevential diagnosis of frimary stuttering and normal nonfluency in children referring to Saba Clinic (Persian)]. Archives of Rehabilitation. 2003; 4(3):45-8.

[37] Forutan E. [The comparison of voice onset time (VOT) of stutters and nonstutters (Persian)]. Journal of Audiology. 2001; 9(1):81-7.

[38] Hillman RE, Gilbert HR. Voice onset time for voiceless stop consonants in the fluent reading of stutterers and nonstutterers. Journal of the Acoustical Society of America. 1977; 61(2):610-1. doi: 10.1121/1.381308

[39] Shaker Ardakani M, Soleymani Z, Torabinejad F, Khoddami SM, Alipour Heydari M. [Fundamental frequency, jitter, and shimmer of adult stutters and nonstutters voice (Persian)]. Audiology. 2007; 16(2):46-50.

[40] Nikkhah S, Shafiei B. [Determination and comparison of reaction time of phonation in stutterers and non-stutterers in two group of age 10-20, 20-30 (Persian)]. Journal of Research in Rehabilitation Sciences. 2013; 9(4):742-753.

[41] Sundara M, Polka L, Baum S. Production of coronal stops by simultaneous bilingual adults. Bilingualism: Language and Cognition. 2006; 9(1):97-114. doi: 10.1017/s1366728905002403

[42] Yang J. Acoustic properties of vowel production in Mandarin-English bilingual and corresponding monolingual chil- dren. $\mathrm{PhD}$ dissertation] Columbus: Ohio State University; 2014.

[43] Kehoe MM, Lleó C, Rakow M. Voice onset time in bilingual German-Spanish children. Bilingualism: Language and Cognition. 2004; 7(1):71-88. doi: 10.1017/s1366728904001282

[44] Chionidou A, Nicolaidis K. Voice onset time in bilingual Greek-German children. Paper presented at: The $18^{\text {th }}$ International Congress of Phonetic Sciences; 2015 Aug 10-14; Glasgow, Scotland.

[45] Khattab G. VOT production in English and Arabic bilingual and monolingual children. Amsterdam Studies in the Theory and History of Linguistic Science Series 4. 2002; 95-122. doi: 10.1075/cilt.230.03kha

[46] Herbst LE. The influence of language dominance on bilingual VOT: A case study. In: $4^{\text {th }}$ University of Cambridge Postgraduate Conference on Language Research (pp. 91-98). Cambridge: Cambridge University Press; 2006.

[47] Healey EC, Gutkin B. Analysis of stutterers' voice onset times and fundamental frequency contours during fluency. Journal of Speech, Language, and Hearing Research. 1984; 27(2):219-25. doi: $10.1044 /$ jshr.2702.219

[48] Robb M, Blomgren M, Chen Y. Formant frequency fluctuation in stuttering and nonstuttering adults. Journal of fluency disorders. 1998; 23(1):73-84. doi: 10.1016/s0094-730x(97)00029-6

[49] Sacco PR, Metz DE. Changes in stutterers' fundamental frequency contours following therapy. Journal of fluency disorders. 1987; 12(1):1-8. doi: 10.1016/0094-730x(87)90035-0

[50] Falck FJ, Lawler PS, Yonovitz A. Effects of stuttering on fundamental frequency. Journal of Fluency Disorders. 1985; 10(2):123-35. doi: 10.1016/0094-730x(85)90020-8

[51] Sacco PR, Metz DE. Comparison of period-by-period fundamental frequency of stutterers and nonstutterers over repeated utterances. Journal of Speech, Language, and Hearing Research. 1989; 32(2):439-44. doi: 10.1044/jshr.3202.439

[52] Hall KD, Yairi E. Fundamental frequency, jitter, and shimmer in preschoolers who stutter. Journal of Speech, Language, and Hearing Research. 1992; 35(5):1002-8. doi: 10.1044/ jshr.3505.1002

[53] Metz DE, Conture EG, Caruso A. Voice onset time, frication, and aspiration during stutterer's fluent speech. Journal of Speech, Language, and Hearing Research. 1979; 22(3):649-56. doi: $10.1044 /$ jshr.2203.649

[54] Howell P, Sackin S, Rustin L. Comparison of speech amotor development in stutterers and fluent speakers between 7 and 12 years old. Journal of Fluency Disorders. 1995; 20(3):243-55. doi: 10.1016/0094-730x(94)00011-h

[55] Agnello J, Wingate ME. Some acoustical and physiological aspects of stuttered speech. Journal of the Acoustical Society of America. 1972; 14:479. doi: 10.1121/1.1981988

[56] Sim HS, Zebrowski PM. Acoustic analysis of stuttering children's fluent speech pre- and post-therapy. Korean Journal of Communication Disorders. 1997; 2:119-136. 
[57] Nudelman HB, Herbrich KE, Hoyt BD, Rosenfield DB. A neuroscience model of stuttering. Journal of Fluency Disorders. 1989; 14(6):399-427. doi: 10.1016/0094-730x(89)90028-4

[58] Basi M, Farazi M, Bakhshi E. Evaluation of Effects of Gradual Increase Length and Complexity of Utterance (GILCU) Treatment Method on the Reduction of Dysfluency in SchoolAged Children with Stuttering. Iranian Rehabilitation Journal. 2016; 14(1):59-62. 
